II. Die Rechtssache hat keine grundsätzliche Bedeutung. Weder die Fortbildung des Rechts noch die Sicherung einer einheitlichen Rechtsprechung erfordern eine Entscheidung des Senats aufgrund mündlicher Verhandlung, die auch sonst nicht geboten ist.

https://doi.org/10.1007/s00350-017-4771-1

\section{Anmerkung zu OLG Köln, Hinweisbeschl. v. 11.1.2017 - 5 U 82/16 (LG Köln)}

\section{Volker Großkopf}

Dem Sachverständigenbeweis kommt im medizinischen und pflegerischen Kontext stets eine besondere Bedeutung für den Ausgang des Verfahrens zu. Das Votum des Sachverständigen kann existenzielle Auswirkungen auf die Parteien haben. Die jüngste Verbesserung der Vorschriften zum Sachverständigenrecht ${ }^{1}$ trägt diesem Gewicht Rechnung, indem $\$ 404$ Abs. 2 ZPO nun dem Richter die Möglichkeit einräumt, die Parteien bereits vor der Ernennung zur Person des Sachverständigen zu hören. Neu ist auch, dass $₫ 407$ a Abs. 2 ZPO dem Sachverständigen eine unverzügliche Prüfungspflicht auferlegt, ob ein Grund vorliegt, der geeignet ist, Misstrauen gegen seine Unparteilichkeit zu rechtfertigen. Mit dem Blick auf das Gutachterwesen in Arzt- und Pflegehaftungsprozessen sind diese Novellierungen prinzipiell geeignet das Vertrauen in die Unabhängigkeit und Qualifikation der Sachverständigen zu stärken.

Erforderlich ist allerdings, dass diese neuen Instrumentarien in der Praxis auch Beachtung und Anwendung finden. Wenngleich die Mehrzahl der Haftungsfälle ganz oder überwiegend medizinischer Natur ist, steigen die Fallzahlen im Bereich der Pflegehaftung. Im Gegensatz zu den spezialisierten Spruchkörpern für Arzthaftungsfälle gibt es jedoch keine auf Pflegefragen spezialisierte Kammern und Senate - was die Pflege für den Juristen zu einer klassischen „Querschnittsmaterie“" macht.

Dabei wäre es, auch im Hinblick auf die zunehmende Bedeutung und die fortschreitende Professionalisierung und Spezialisierung dieses Berufsfeldes, geboten, dem Thema von Seiten der Justiz gleichsam mehr Aufmerksamkeit zu schenken. Schließlich käme kein Richter auf die Idee, ein Gutachten etwa über einen gefäßchirurgischen Sachverhalt an einen Augenarzt zu vergeben. Doch wenn es um die wohl jährlich mehr als 1000 verhandelten Fälle von Pflegefehlern in Deutschland geht, wirken mitunter rein oder überwiegend ärztlich bewanderte Experten in der Urteilsfindung mit. Da stellt sich die Frage: „Kann der Arzt darüber ein Urteil fällen, oder braucht es nicht mehr fachliche Expertise?" Die Antwort fällt aus der Sicht der Pflegewissenschaft eindeutig aus: es ist nicht länger haltbar, dass die Verantwortung für pflegerisches Handeln von den Gerichten überwiegend den Ärzten zugeschrieben wird"

1) Gesetz zur Änderung des Sachverständigenrechts und zur weiteren Änderung des Gesetzes über das Verfahren in Familiensachen und in den Angelegenheiten der freiwilligen Gerichtsbarkeit sowie zur Änderung des Sozialgerichtsgesetzes, der Verwaltungsgerichtsordnung, der Finanzgerichtsordnung und des Gerichtskostengesetzes v. 11. 10.2016 (BGBl. I S. 2222).

2) Ursula Laag: Begutachtung von Pflegefehlern: (K)eine Aufgabe der Ärzte. Rechtsdepesche für das Gesundheitswesen (RDG) Nr. 3/2013 (Mai/Jun), S. 116.

Professor Dr. iur. Volker Großkopf,

Geschäftsführer PWG-Seminare, Geschäftsführung G \& S Verlag, Salierring 48, 50677 Köln, Deutschland

\section{Behandlungsfehler durch Aufrechterhaltung der künstlichen Ernährung mittels PEG-Sonde}

\author{
BGB §§ 1901 a ff.; StGB §223
}

1. Die medizinische Indikation kann auch in solchen Fällen fehlen bzw. wegfallen, in denen die lebenserhaltende Maßnahme nur mehr Leiden verlängert. Anders als das scheinbar objektive Kriterium der ,unmittelbaren Todesnähe" ermöglicht eine sorgfältige einzelfallbezogene Abwägung im Rahmen der Indikationsstellung einen umfassenden Schutz des Patienten.

2. Kann mit der künstlichen Ernährung (PEG-Sonde) kein Therapieziel mehr verfolgt werden, das über die reine Aufrechterhaltung des Lebens um die Dauer eben dieser Maßnahme hinausgeht, trifft den behandelnden Arzt nach $₫ 1901$ b Abs. 1 BGB die Pflicht zur Konsultation des Betreuers. Die Verletzung dieser Pflicht begründet einen Behandlungsfehler.

3. Die Feststellung, dass dieser Behandlungsfehler ursächlich geworden ist für die Verlängerung des rechtfertigungsbedürftigen körperlichen Eingriffs, erfordert allerdings den Nachweis, dass im Falle einer Erörterung der Situation zwischen Arzt und Betreuer tatsächlich die Entscheidung zugunsten einer Beendigung der PEG-Ernährung getroffen worden wäre.

4. Ob ein Leben ohne Aussicht auf Besserung erhalten oder eine lebenserhaltende Versorgung eingestellt


zu entscheiden. Dabei kommt dem Schutz menschlichen Lebens im Zweifel Vorrang zu. (Leitsätze des Bearbeiters)

LG München I, Urt. v. 28.11.2016 - 9 O 5246/14

Problemstellung: Die Schadensersatzklage des Sohnes und gleichzeitigen Erblassers (aber nicht vormaligen Betreuers) betrifft die Fortführung der PEGErnährung eines schwerkranken Pflegeheimbewohners, ohne dass der zuständige Hausarzt die Indikation hierfür fortlaufend aufs Neue geprüft und den Eingriff rechtzeitig beendet hätte. Im Ausgangspunkt betont die Kammer die doppelte Rechtfertigungsbedürftigkeit nicht nur der Einleitung, sondern gleichermaßen der Fortführung eines - auch lebenserhaltenden - ärztlichen Heileingriffs. Der Indikation komme daher ,,zunächst eine weichenstellende Funktion“ zu, weil sich ,die Frage nach der Einwilligung ... erst dann [stellt], wenn und soweit die Indikation gegeben ist“. Deshalb ,kann regelmäßig eine nicht indizierte Behandlung weder durch eine Patientenverfügung noch durch einen an Stelle des Patienten berufenen Entscheidungsträger angeordnet werden“.

Obgleich die Kammer für die vorliegende Fallkonstellation, sachverständig durch einen Allgemeinmediziner beraten, jedenfalls ab Beginn des Jahres 2010 keine Indikation mehr für die PEG-Sonden-Ernährung erkennt, soll dies den behandelnden Arzt nicht zwangsläufig zur Einstellung der künstlichen Ernährung zwingen: Für diese Situation, die sich von der erstmaligen Anlage einer PEG-Sonde unterscheide, habe der Gesetzgeber mit den $\$ 1901$ a ff. BGB eine ausdrückliche, vorrangige Regelung getroffen, die - s. \$1901b Abs. 1 BGB - dem behandelnden Arzt ,gerade nicht den eigenverantwortlichen Abbruch der Behandlung auferleg[e], sondern vielmehr die Verpflichtung, [...] mit dem Betreuer die Fortsetzung der Maßnahme zu erörtern“. Behandlungs-

Eingesandt und bearbeitet von Prof. Dr. iur. Gunnar Duttge, Institut für Kriminalwissenschaften/Zentrum für Medizinrecht, Georg-August-Universität,

Platz der Göttinger Sieben 6, 37073 Göttingen, Deutschland 